\title{
Errata to Volume 3, pages 225-259 regarding the article
}

On the first boundary value problem for the classical theory of elasticity in a three-dimensional domain with a singular boundary

by PIERANITA CASTELLANI RIZZONELLI

p. 2256 th, 7 th and 8 th rows from the bottom

Errata: This means that it is not even known, a priori, that surface summability of these functions, is a necessary condition for the validity of the above mentioned Betti and Clapeyron theorems.

Corrige: This means that the surface summability of these functions is not even known, a priori. This summability is a necessary condition for the validity of the above mentioned Betti and Clapeyron theorems.

p. 228 14th row from above

Errata: by: $\xi \in \Gamma^{s}$

Corrige: by: $\xi \in \Gamma_{s}$

p. 228 15th row from above

Errata: between $\Gamma^{s}$ and

Corrige: between $\Gamma_{s}$ and

p. 236 1st row from the bottom

Errata: follows from (15), (21) and (22) gives (6).

Corrige: we have that inequality (6) follows from (15), (21) and (22).

p. 2449 th row from the bottom

Errata: $a_{\varepsilon}$ can be chosen independent at $\xi$.

Corrige: $a_{\varepsilon}$ can be chosen independent of $\xi$. 